\title{
Crystallized half-skyrmions and inverted half-skyrmions in the condensation of spin-1 Bose gases with spin-orbit coupling
}

\author{
S.-W. Su, ${ }^{1}$ I.-K. Liu, ${ }^{2}$ Y.-C. Tsai, ${ }^{3}$ W. M. Liu, ${ }^{4}$ and S.-C. Gou ${ }^{2, *}$ \\ ${ }^{1}$ Department of Physics, National Tsing Hua University, Hsinchu 30013, Taiwan \\ ${ }^{2}$ Department of Physics, National Changhua University of Education, Changhua 50058, Taiwan \\ ${ }^{3}$ Department of Photonics, Feng Chia University, Taichung 40724, Taiwan \\ ${ }^{4}$ Instuite of Physics, Chinese Academy of Sciences, Beijing 100190, People's Republic of China \\ (Received 18 December 2011; revised manuscript received 2 April 2012; published 2 August 2012)
}

\begin{abstract}
The nonequilibrium dynamics of a rapidly quenched spin-1 Bose gas with spin-orbit coupling is studied. By solving the stochastic projected Gross-Pitaevskii equation, we show that crystallization of half-skyrmions (merons), can occur in a spinor condensate of ${ }^{87} \mathrm{Rb}$. The stability of such a crystal structure is analyzed. Likewise, inverted half-skyrmions can be created in a spin-polarized spinor condensate of ${ }^{23} \mathrm{Na}$. Our studies provide a chance to explore the fundamental properties of skyrmionlike matter.
\end{abstract}

DOI: 10.1103/PhysRevA.86.023601

PACS number(s): 67.85.Fg, 03.75.Kk, 03.75.Lm, 03.75.Mn

\section{INTRODUCTION}

Spin-orbit coupling (SOC) [1] is a ubiquitous quantum phenomenon which links the internal (spin) and orbital (linear or angular momentum) degrees of freedom of a particle. The best-known example of SOC arises in the motion of electrons in the atom, where the electron's orbiting around the nucleus can affect the orientation of electron's spin. Recently, SOC with tunable strength has been realized by Lin et al. [2] by irradiating two laser beams intersecting in a pseudo-spin- $\frac{1}{2}$ Bose-Einstein condensate (BEC) of ${ }^{87} \mathrm{Rb}$ [3]. The lasers are detuned from Raman resonance so that the momentum and spin can couple via exchanging photons in the two beams. This opens new possibilities to simulate the role of SOC for a wide range of phenomena in condensed-matter physics by using ultracold atoms and promises applications to quantum computing [4], spintronics [5,6], and topological insulators [7-9]. Inspired by the experimental realization in Ref. [2], theoretical extensions using atomic BECs have been studied by a number of authors [10-13]. These include the direct incorporation of SOC into the spin- $-\frac{1}{2}, 1$, and 2 BECs, in which nontrivial ground-state structures have been predicted. On the other hand, new types of excitations, such as the Rashba pairing bound state (Rashbon) [14,15], have also been predicted in degenerate fermionic gases with SOC [16].

So far, most of the theoretical studies on the ultracold atoms with SOC were focused in the limit of zero temperature. It is of fundamental importance to see how the nonlocal nature of SOC affects the pattern of spontaneous symmetry breaking. In this work, we study the nonequilibrium dynamics during the condensation of a spin-1 Bose gas with SOC. In particular, we focus on the formation of topological defects in the limit of rapid temperature quench. According to the Kibble-Zurek mechanism $[17,18]$, topological defects can be created via phase transitions at finite temperatures, which are caused by spontaneous symmetry breaking and thermal fluctuations near the critical point $[19,20]$. By solving the stochastic projected Gross-Pitaevskii equation (SPGPE), we show that, in a spin-1 $\mathrm{BEC}$, the combination of SOC, spin-exchange interaction, and

\footnotetext{
*scgou@cc.ncue.edu.tw
}

thermal fluctuations can generate half-skyrmion-like excitations. The half-skyrmions (merons) are a peculiar topological defect that was originally hypothesized as the half-instanton in particle physics [21] and later the half-skyrmion in the quantum Hall systems [22,23]. Since merons carry half a unit of topological charge, it is believed that the isolated merons can be observed only when particular boundary conditions are imposed. By far, merons have been created in the superfluid ${ }^{3} \mathrm{He}-A$ in a rotating cylinder $[24,25]$ and the spinor BEC with a constraining magnetic field [26]. In what follows, we show that stable collective excitations such as the crystalline order of merons and other isolated variants can be created in a rapidly quenched spinor BEC with SOC.

The organization of this paper is as follows. In Sec. II, an account of SPGPE for the spin-orbit coupled spin-1 BEC is given. The numerical results for the spin-orbit coupled spin-1 $\mathrm{BEC}$ with ferromagnetic and antiferromagnetic interaction are presented, respectively, in Sec. III. The structures of the spin textures for the emergent topological defects are discussed. In particular, the stability of the crystallized merons is analyzed. Finally, some concluding remarks are presented in Sec. IV, including the scheme for fulfilling the rapid temperature quench.

\section{THEORETICAL MODEL}

The order parameter of a spin-1 BEC is given by $\Psi=\left(\Psi_{1}, \Psi_{0}, \Psi_{-1}\right)^{\mathrm{T}}$, where the superscript $\mathrm{T}$ stands for the transpose and $\Psi_{m_{F}}\left(m_{F}= \pm 1,0\right)$ denotes the macroscopic wave function of the atoms condensed in the spin state $\left|1, m_{F}\right\rangle$. The total particle number, $N$, and total magnetization, $M$, are determined by $\int|\Psi|^{2} d^{3} r=N$, and $\int\left(\left|\Psi_{1}\right|^{2}-\left|\Psi_{-1}\right|^{2}\right) d^{3} r=$ $M$. In the following, we consider the Dresselhaus type of SOC, $H_{s o}=\sum_{\alpha} V_{\alpha} p_{\alpha} F_{\alpha}$, where $V_{\alpha}, p_{\alpha}$, and $F_{\alpha}$ are, respectively, the coupling strength, the linear momentum, and the $3 \times 3$ matrix of the spin-1 angular momentum in the $\alpha(=x, y, z)$ direction. Such a SOC can be realized, in principle, by extending the experimental methods in [2]. However, in lifting the degeneracy of the hyperfine spin states, a weaker magnetic field is needed to avoid the decoupling of spin states due to quadratic Zeeman shift. We notice that a generalized 
Rashba-Dresselhuas type of SOC for spin-1 particles has been proposed by using a tetrapod configuration $[27,28]$. Nonetheless, the atomic setup in [27] is beyond the scope of the current studies, as the spin-exchange interaction characterizing the spinor BEC may not be properly manifested in such a scheme.

In the absence of magnetic field, the dynamics of $\Psi$ is described by the following coupled nonlinear Schrödinger equation:

$$
\begin{aligned}
i \hbar \frac{\partial}{\partial t} \Psi_{j}= & H_{j}^{G P} \Psi_{j}=\mathcal{H} \Psi_{j}+g_{s} \sum_{\alpha} \sum_{n, k, l}\left(F_{\alpha}\right)_{j n} \Psi_{n} \Psi_{k}^{*}\left(F_{\alpha}\right)_{k l} \Psi_{l} \\
& +\frac{\hbar}{i} \sum_{\alpha} \sum_{n} V_{\alpha}\left(F_{\alpha}\right)_{j n} \partial_{\alpha} \Psi_{n} \quad(j, k, l, n=-1,0,1) .
\end{aligned}
$$

Here $\mathcal{H}=-\hbar^{2} \nabla^{2} / 2 m+U(\mathbf{r})+g_{n}|\Psi|^{2}$ denotes the spinindependent part of the Hamiltonian, with $U(\mathbf{r})$ being the trapping potential. The coupling constants $g_{n}$ and $g_{s}$ characterizing the density-density and spin-exchange interactions, respectively, are related to the $s$-wave scattering lengths $a_{0}$ and $a_{2}$ in the total spin channels $F_{\text {total }}=0,2$ by $g_{n}=$ $4 \pi \hbar^{2}\left(a_{0}+2 a_{2}\right) / 3 m, g_{s}=4 \pi \hbar^{2}\left(a_{2}-a_{0}\right) / 3 m$ [29,30]. Note that $g_{n}$ is always positive, yet $g_{s}$ can be either positive or negative. A spin-1 BEC is said to be ferromagnetic (FM) when $g_{s}<0$ and antiferromagnetic (AFM) when $g_{s}>0$. As we focus on the dynamics of spin texture, we introduce the basis set $\Psi_{\alpha}(\alpha=x, y, z)$, such that $\Psi_{ \pm 1}=\left( \pm \Psi_{x}+i \Psi_{y}\right) / \sqrt{2}$ and $\Psi_{0}=\Psi_{z}$. It follows that the new basis states satisfy $F_{\alpha}|\alpha\rangle=0$, and the spin texture, which is parallel to the local magnetic moment, can be defined by $\mathbf{S}(\mathbf{r})=i \tilde{\Psi}^{\dagger} \times \tilde{\Psi} /|\Psi|^{2}=|S(\mathbf{r})| \hat{\mathbf{s}}$, where $\tilde{\Psi}=\left(\Psi_{x}, \Psi_{y}, \Psi_{z}\right)^{\mathrm{T}}[31]$, and $\hat{\mathbf{s}}(\mathbf{r})$ is the local unit vector.

The dynamics of a BEC at nonzero temperatures can be described by the SPGPE [32-34], which is based on the assumption that the system can be treated as a condensate band in contact with a thermal reservoir comprising of all noncondensed particles. The condensate band is described by the truncated Wigner method [35] including the projected $c$-field method, while the noncondensate band is by the quantum kinetic theory [36,37]. A direct generalization to the spinor BECs with SOC leads to the following set of coupled SPGPEs [38]:

$$
d \Psi_{j}=\mathcal{P}\left\{-\frac{i}{\hbar} \hat{H}_{j}^{G P} \Psi_{j}+\frac{\gamma_{j}}{k_{B} T}\left(\mu-\hat{H}_{j}^{G P}\right) \Psi_{j}\right\} d t+d W_{j},
$$

where $T$ and $\mu$ denote the final temperature and chemical potential, $\gamma_{j}$ is the growth rate for the $j$ th component, and $d W_{j}$ is the complex-valued white noise associated with the condensate growth, which is characterized by the fluctuation-dissipation relation $\left\langle d W_{i}^{*}(\mathbf{x}, t) d W_{j}\left(\mathbf{x}^{\prime}, t\right)\right\rangle=2 \gamma_{j} \delta_{i j} \delta_{C}\left(\mathbf{x}-\mathbf{x}^{\prime}\right) d t$, where $\delta_{C}$ is the Dirac $\delta$ function for the condensate band field [32]. Note that the projection operator $\mathcal{P}$ restricts the dynamics of
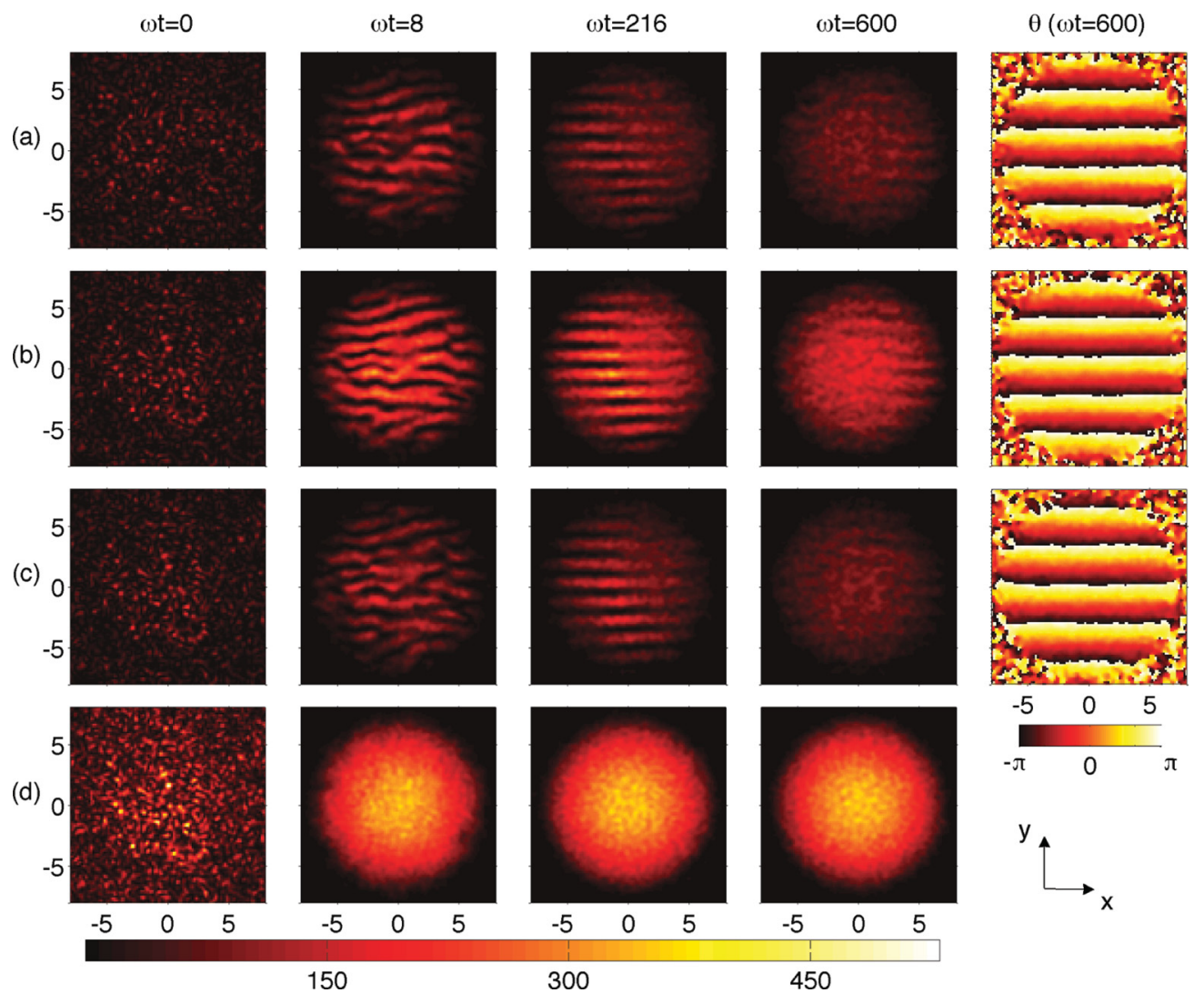

150



FIG. 1. (Color online) Snapshots of the density profiles, (a) $\left|\Psi_{-1}\right|^{2}$, (b) $\left|\Psi_{0}\right|^{2}$, (c) $\left|\Psi_{1}\right|^{2}$, and (d) $|\Psi|^{2}$, of a spinor BEC of ${ }^{87}$ Rb during quench. The strengths of the SOC are $V_{x}=1, V_{y}=2$ and the final temperature is $k_{B} T=2$. The axes of directions are indicated in the bottom right corner. The rightmost column shows the phase profile of equilibrium state. The final particle numbers are $N_{ \pm 1} \approx 7.44 \times 10^{3}, N_{0} \approx 1.45 \times 10^{4}$. 
the spinor BEC in the lower energy region below the cutoff energy $E_{R}$.

The numerical procedures for solving SPGPEs are described as follows. First, the initial state of each $\Psi_{j}$ is sampled by using the grand-canonical ensemble for an ideal spin-1 Bose gas characterized by chemical potentials $\mu_{j, 0}$ at a temperature $T_{0}$ below the critical temperature. The spatial dependence of the initial state can be specified as a linear combination of plane waves with discretized momentum $\mathbf{k}=2 \pi\left(n_{x}, n_{y}\right) / L\left(n_{x}, n_{y}\right.$ $\in Z$ and $L$ is the size of the system); that is, $\Psi_{j}(t=0)=$ $\sum_{\mathbf{k}}^{E_{R}} a_{j, \mathbf{k}} \psi_{\mathbf{k}}(\mathbf{r})$, where $\psi_{\mathbf{k}}(\mathbf{r})$ are the plane-wave solutions. The condensate band lies below the energy cutoff $E_{R}>E_{\mathrm{k}}=$ $\hbar^{2}|\mathbf{k}|^{2} / 2 m$. The distribution is specified by $a_{j, \mathbf{k}}=\left(N_{j, \mathbf{k}}+\right.$ $1 / 2)^{1 / 2} \eta_{j, \mathbf{k}}$, where $N_{j, \mathbf{k}}=\left\{\exp \left[\left(E_{j, \mathbf{k}}-\mu_{j .0}\right) / k_{B} T_{0}\right]-1\right\}^{-1}$ and $\eta_{j, \mathbf{k}}$ are the complex Gaussian random variables with moments $\left\langle\eta_{j, \mathbf{k}} \eta_{j, \mathbf{k}^{\prime}}\right\rangle=\left\langle\eta_{j, \mathbf{k}}^{*} \eta_{j, \mathbf{k}^{\prime}}^{*}\right\rangle=0$ and $\left\langle\eta_{j, \mathbf{k}} \eta_{j, \mathbf{k}^{\prime}}^{*}\right\rangle=\delta_{\mathbf{k k}^{\prime}}$. Second, to simulate the thermal quench, we let the SPGPEs evolve for a short while by keeping $T=T_{0}, \mu=\mu_{j, 0}$ for all $j$ to obtain a high temperature initial thermalized state, and then alter the temperature and chemical potential to the given final values $T<T_{0}$ and $\mu>\mu_{j, 0}$. For simplicity, we shall consider a two-dimensional isotropic trap, $U(\mathbf{r})=m \omega^{2}\left(x^{2}+y^{2}\right) / 2$, such that the length, time, and energy can be respectively scaled in units of $\sqrt{\hbar / m \omega}, \omega^{-1}$, and $\hbar \omega$ in our simulations.

\section{RESULTS AND DISCUSSIONS}

We first study the condensation of ${ }^{87} \mathrm{Rb}\left(g_{s}<0\right)$ in the presence of SOC. The initial state is sampled at $k_{B} T_{0}=500$, $\mu_{j, 0}=2$ and we set $E_{R}=50.5, \mu=25, \hbar \gamma_{j} / k_{B} T=0.05$ $(j= \pm 1,0)$ for two final temperatures, $k_{B} T=2,10$. In this paper, we consider the in-plane coupling, that is, $V_{x}, V_{y} \neq 0$ and $V_{z}=0$ for a two-dimensional system of finite size. The domain of computation has a dimension of $20.05 \times 20.05$ and is discretized by a square grid of $128 \times 128$ points on the $x y$ plane with lattice spacing $\Delta x=\Delta y \approx 0.157$. In the experiment of [2], the SOC strength can be tuned by varying the wavelength of the laser field and the angle between the two laser beams. For example, for two laser beams with wavelength $\lambda=804.1 \mathrm{~nm}$ intersecting with an angle of $\pi / 12$, the corresponding SOC strength is 1.25 in the dimensionless unit and when the intersecting angle is changed to $\pi / 6$, the corresponding SOC strength becomes 3.08. In the following simulations, we begin with the condition of $\left|V_{x}\right| \neq\left|V_{y}\right|$. In Fig. 1, the stripe structures develop presently in the density and phase profiles of each component during condensation. The stripes in the density profiles fade away gradually while the system approaches equilibrium. On the other hand, the stripe structures in the phase profiles retain persistently, which are exactly the plane-wave (PW) state discovered in Ref. [11]. The evolution of the spin texture is shown in Fig. 2. Clearly, spin domains form shortly after the quench starts. The domain walls are composed of arrays of spin vortices, which drift outward and disappear eventually. When the system reaches equilibrium in the PW state, the spins align uniformly on the $x y$ plane. The PW state is stable at higher temperatures despite that the density profiles and the spin texture may fluctuate noticeably, as shown in Fig. 3. The PW state appears exclusively when $\left|V_{x}\right| \neq\left|V_{y}\right|$ or $\left|V_{x}\right|=\left|V_{y}\right|=V<0.8$. When $\left|V_{x}\right|=\left|V_{y}\right|=V>0.8$ [39], (a) $\omega t=0$

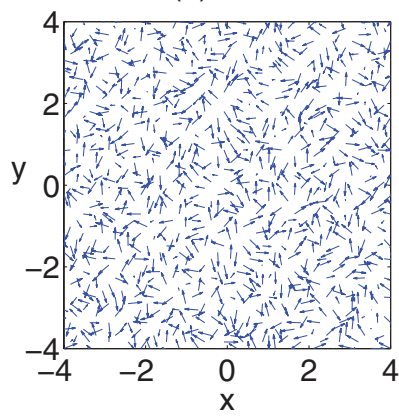

(c) $\omega t=300$

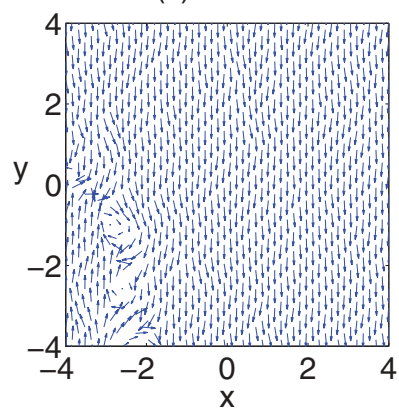

(b) $\omega t=156$

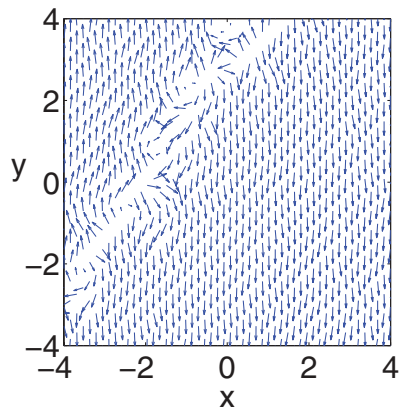

(d) $\omega t=600$

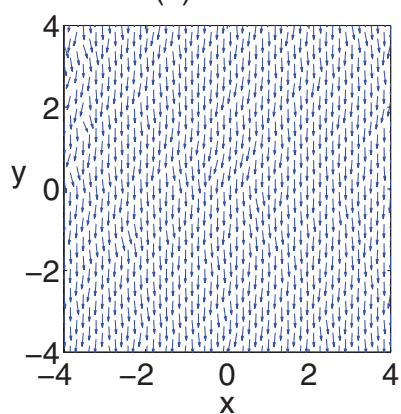

FIG. 2. (Color online) Snapshots of the $\hat{\mathbf{s}}$ texture caused by the results shown in Fig. 1. Arrays of spin vortices (merons) of same handedness appear as domain walls in (b), which drift away towards the periphery of the condensate as shown in (c) and (d).

periodic structures, which are caused by the formation of grids of dark soliton in $\Psi_{ \pm 1}$ and vortex lattice in $\Psi_{0}$, may appear in the equilibrium density profiles of all components (a)

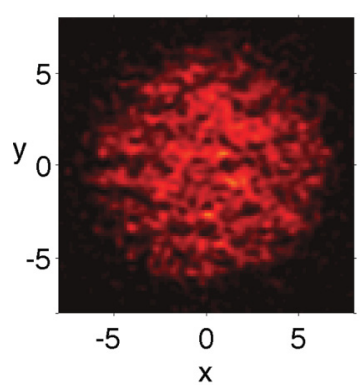

(c)

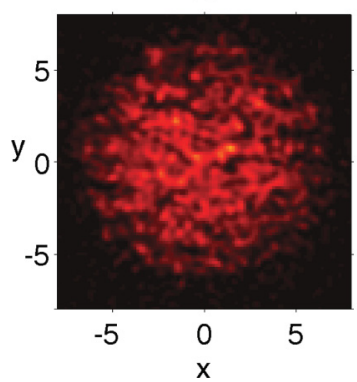

(b)

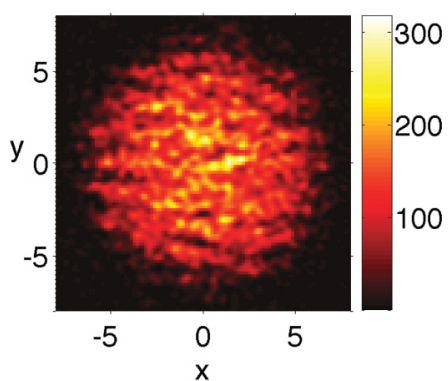

(d)

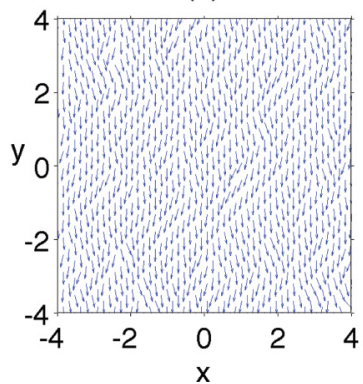

FIG. 3. (Color online) The equilibrium density profiles, (a) $\left|\Psi_{-1}\right|^{2}$, (b) $\left|\Psi_{0}\right|^{2}$, (c) $\left|\Psi_{1}\right|^{2}$, and (d) the $\hat{\mathbf{s}}$ texture, of the spinor BEC of ${ }^{87} \mathrm{Rb}$ at $k_{B} T=10$. The strengths of the SOC are $V_{x}=1, V_{y}=2$. The final particle numbers are $N_{ \pm 1} \approx 7.95 \times 10^{3}, N_{0} \approx 1.46 \times 10^{4}$. 

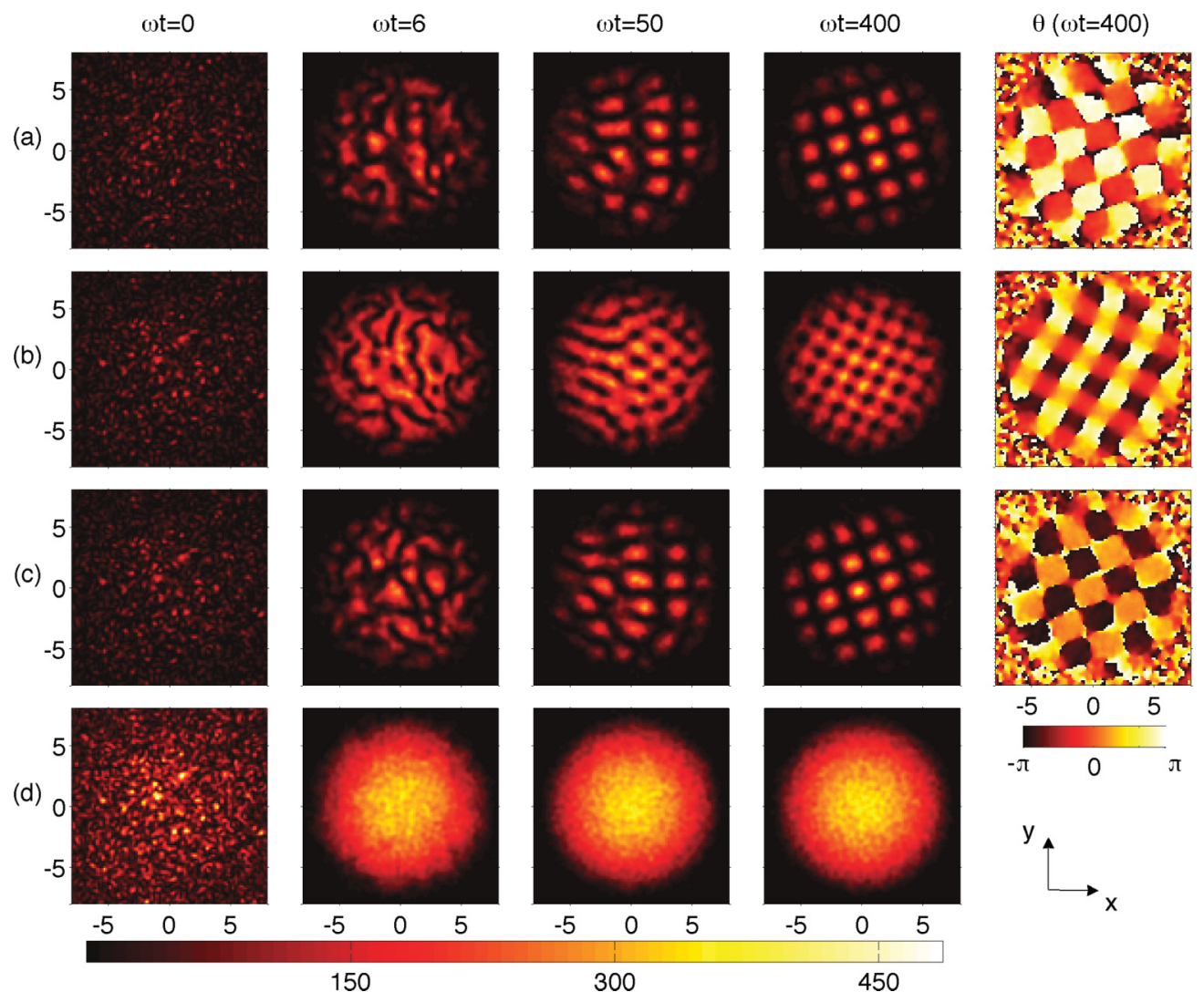

FIG. 4. (Color online) Snapshots of the density profiles, (a) $\left|\Psi_{-1}\right|^{2}$, (b) $\left|\Psi_{0}\right|^{2}$, (c) $\left|\Psi_{1}\right|^{2}$, and (d) $|\Psi|^{2}$, of a spinor BEC of ${ }^{87} \mathrm{Rb}$ during quench. The strengths of the SOC are $V_{x}=V_{y}=V=1.8$ and the final temperature is $k_{B} T=2$. The axes of directions are indicated in the bottom right corner. The rightmost column shows the phase profiles of equilibrium state. The final particle numbers are $N_{ \pm 1} \approx 7.21 \times 10^{3}$, $N_{0} \approx 1.42 \times 10^{4}$.

as shown in Fig. 4. We first consider the case of $k_{B} T=2$, as shown in Fig. 5(a), where the spin texture consists of two interlacing square lattices of spin vortex with opposite vorticities. Such a configuration will be denoted as the spin vortex lattice (SVL) state. When $V>0.8$, the simulations will end up in the equilibrium configuration of either PW or SVL


FIG. 5. (Color online) (a) The three-dimensional orientation for the equilibrium $\hat{\mathbf{s}}$ texture associated with the results of Fig. 4 . The color of the arrow indicates the magnitude of $s_{z}$. (b) The topological charge density $\sigma$ of the equilibrium spin texture. (c) The associated spin-density distribution $|\mathbf{S}(\mathbf{r})|$. states. However, the probability for the appearance of SVL state decreases as $V$ increases. When $V>4$, the SVL state can scarcely appear in the simulations. Comparing the energies of PW and SVL states from the numerical results, the SVL state is shown to have a higher energy than the PW state. In the SVL state, all spin vortices exhibit similar structure: The central spin always points to the $z$ axis, while the others increasingly tilt and finally lie on the $x y$ plane, forming a circulation pattern (either left-handed or right-handed) away from the center. The topological charge density, $\sigma=\mathbf{s} \cdot\left(\partial_{x} \mathbf{s} \times \partial_{y} \mathbf{s}\right) / 4 \pi$, is plotted in Fig. 5(b) and the vortices with left (right)-handed circulation correspond to a positive (negative) topological charge density. This is exactly the Mermin-Ho vortex [40-42], or meron [43]. Integrating $\sigma$ over the primitive unit cell, we identify that $Q=\int_{\text {unit cell }} \sigma d^{2} r= \pm 1 / 2$, which corresponds to the merons and antimerons, respectively. Due to the FM nature of the condensate, a meron and its neighboring counterpart will pair up to form vortex-dipole so as to lower the energy. The meron-antimeron pair has been predicted as the lowest energy excitation in the bilayer quantum Hall systems [23]. Since meron and antimeron are simply mirror images of each other, we do not distinguish them in the following paragraphs unless their distinction needs to be explicitly addressed.

Alternatively, the SVL state can be visualized as the mosaic of vortex-quadrupoles consisting of four mutually adjoining merons and antimerons [see Fig. 5(a)]. From Fig. 5(c), we find that the spin density $|\mathbf{S}(\mathbf{r})|$ vanishes in the center of 
(a)

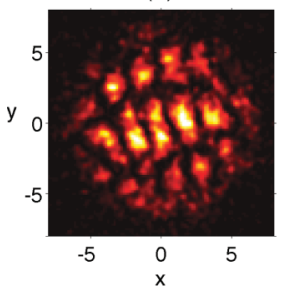

(b)



(c)

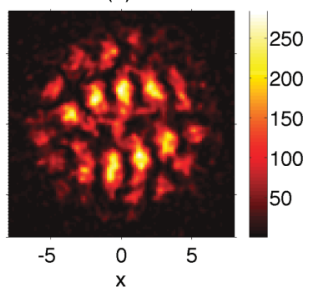

FIG. 6. (Color online) The equilibrium density profiles, (a) $\left|\Psi_{-1}\right|^{2}$, (b) $\left|\Psi_{0}\right|^{2}$, and (c) $\left|\Psi_{1}\right|^{2}$, of a spinor BEC of ${ }^{87} \mathrm{Rb}$ at $k_{B} T=10$ with $V_{x}=V_{y}=V=1.8$. The densities of all components fluctuate considerably in comparison with those in the lower-temperature case shown in Fig. 4. The final particle numbers are $N_{ \pm 1} \approx 7.66 \times 10^{3}$, $N_{0} \approx 1.41 \times 10^{4}$.

each vortex-quadrupole, indicating that a polar-core vortex is located thereat. Hence, the SVL state is actually consisted of interlacing lattices of Mermin-Ho vortices and polar-core spin vortices. We notice that the formation of the SVL state with merons and polar-core spin vortices has been predicted for a highly polarized and fast-rotating FM spinor BEC [31]. Nonetheless, the SVL states in both cases are of different essence. In [31], the SVL state arises as the lowest-energy state under rotation at zero temperature while the one in our case is the result of a SOC and Kibble-Zurek mechanism. Moreover, the latter is a metastable excited state, which marks an evidence for the importance of nonequilibrium dynamics. In the case of the higher final temperature, $k_{B} T=10$, the thermal fluctuations distort the lattice structures of all components as well as the magnetization of the condensate, as shown in Figs. 6 and 7 , respectively. It can be clearly seen from the topological charge density plotted in Fig. 7(b) that the crystalline order of the merons is more vulnerable to the thermal fluctuations, which dissolves at higher temperatures.

To gain more insight into the SVL state, it is constructive to examine the properties of SVL states in momentum space. In Fig. 8, the equilibrium density $\left|\Psi_{0}(\mathbf{p})\right|^{2}$ is plotted for SVL states at different temperatures. The other two density profiles, $\left|\Psi_{ \pm 1}(\mathbf{p})\right|^{2}$, are not plotted for they have the same pattern

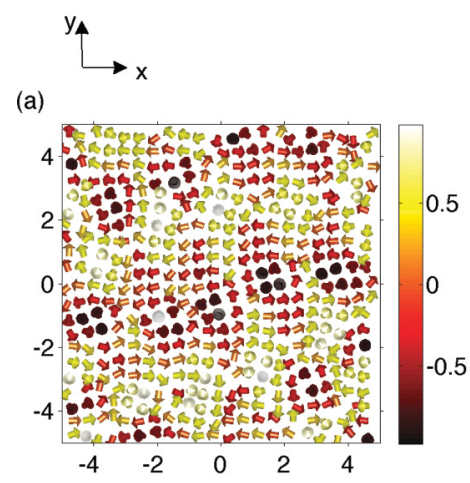

(b)

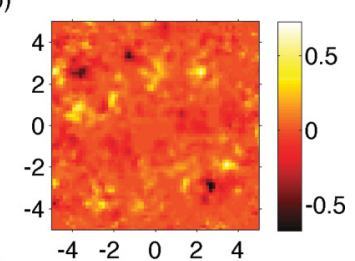

(c)

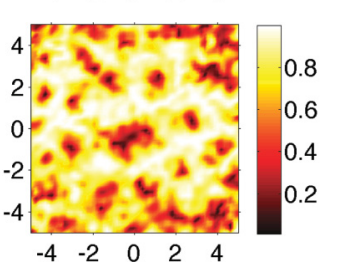

FIG. 7. (Color online) (a) The three-dimensional orientation for the equilibrium $\hat{\mathbf{s}}$ texture associated with the results of Fig. 6 . The color of the arrow indicate the magnitude of $s_{z}$. (b) The topological charge density $\sigma$ of the equilibrium spin texture. (c) The associated spin-density distribution $|\mathbf{S}(\mathbf{r})|$.
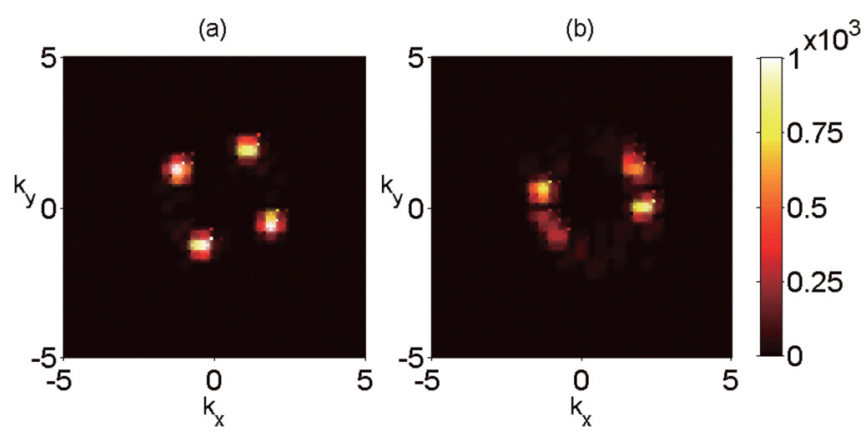

FIG. 8. (Color online) The corresponding equilibrium density profiles in momentum space for the SVL states shown in (a) Fig. 4 and (b) Fig. 6, respectively.

as that of $\left|\Psi_{0}(\mathbf{p})\right|^{2}$. We find that the density distribution is sharply peaked at $\mathbf{p} \approx \pm 1.76 \mathbf{e}_{x}, \pm 1.76 \mathbf{e}_{y}$ at lower temperature. This suggests that the equilibrium state might consist of four PWs with $\mathbf{p}= \pm \mathbf{q}, \pm \mathbf{q}^{\prime}$, where $\mathbf{q} \cdot \mathbf{q}^{\prime}=0$ and $|\mathbf{q}|=\left|\mathbf{q}^{\prime}\right|$. The counterpropagating modes with $\mathbf{p}= \pm \mathbf{q}, \pm \mathbf{q}^{\prime}$ form two orthogonal standing waves that superimpose to generate the periodic structures in all density profiles in Fig. 3. In the limit of weak trapping, it was shown that the one-particle Hamiltonian, $p^{2} / 2 m+V \mathbf{p} \cdot \mathbf{F}$, is minimized by $|\mathbf{p}|=V[11,12]$. This agrees with the aforementioned numerical result, in which $|\mathbf{p}|=1.76 \approx V=1.8$. As a result,

$$
\begin{aligned}
\Psi_{ \pm 1}(\mathbf{p})= & \frac{\sqrt{N} e^{\mp i \theta}}{4}\left[-\delta^{(2)}\left(\mathbf{p}-\frac{V \mathbf{q}}{|\mathbf{q}|}\right)-\delta^{(2)}\left(\mathbf{p}+\frac{V \mathbf{q}}{|\mathbf{q}|}\right)\right. \\
& \left.\mp \delta^{(2)}\left(\mathbf{p}-\frac{V \mathbf{q}^{\prime}}{\left|\mathbf{q}^{\prime}\right|}\right) \mp \delta^{(2)}\left(\mathbf{p}+\frac{V \mathbf{q}^{\prime}}{\left|\mathbf{q}^{\prime}\right|}\right)\right],
\end{aligned}
$$

and

$$
\begin{aligned}
\Psi_{0}(\mathbf{p})= & \sqrt{\frac{N}{8}}\left[\delta^{(2)}\left(\mathbf{p}-\frac{V \mathbf{q}}{|\mathbf{q}|}\right)-\delta^{(2)}\left(\mathbf{p}+\frac{V \mathbf{q}}{|\mathbf{q}|}\right)\right. \\
& \left.+i \delta^{(2)}\left(\mathbf{p}-\frac{V \mathbf{q}^{\prime}}{\left|\mathbf{q}^{\prime}\right|}\right)-i \delta^{(2)}\left(\mathbf{p}+\frac{V \mathbf{q}^{\prime}}{\left|\mathbf{q}^{\prime}\right|}\right)\right],
\end{aligned}
$$

where $\theta=\tan ^{-1}\left(q_{y} / q_{x}\right)$, and $\delta^{(2)}$ denotes the two-dimensional Dirac $\delta$ function. Consequently, we obtain

$$
\begin{array}{r}
\mathbf{S}(\mathbf{r})=(\cos \theta \cos u \sin v+\sin \theta \sin u \cos v, \\
\cos \theta \sin u \cos v-\sin \theta \cos u \sin v, \cos u \cos v),
\end{array}
$$

where $\quad u=V(x \cos \theta+y \sin \theta)$ and $v=V(-x \sin \theta+$ $y \cos \theta)$. The above analytical results fairly reproduce the spin texture plotted in Fig. 5(a) in spite of the presence of trapping potential. The meron and polar cores are centered at $\mathbf{r}_{\text {meron/polar }}=R(\theta) \mathbf{d}_{\text {meron/polar }}$, where $R(\theta)$ is the rotation matrix on $x y$ plane, $\mathbf{d}_{\text {meron }}=(n, l) \pi / V$, and $\mathbf{d}_{\text {polar }}=(n+1 / 2, l+$ $1 / 2) \pi / V$, with $n, l$ being integers. It should be noted that the merons are centralized around the vortex cores of $\Psi_{0}$. In our simulations, PW and SVL states appear alternatively, and their probabilities of appearance depend on the magnitude of $V$. Using the imaginary time propagation method, the SVL state is shown to have a higher energy than the PW state. Analytically, the energies of PW and SVL states for a homogeneous spinor BEC can be calculated by making use of Eqs. (3) and (4). As PW and SVL states both yield the same minimized energy for the single-particle Hamiltonian with a fixed $N$, we only 



300

600

900

FIG. 9. (Color online) Snapshots of the equilibrium density profiles, (a) $\left|\Psi_{-1}\right|^{2}$, (b) $\left|\Psi_{0}\right|^{2}$, (c) $\left|\Psi_{1}\right|^{2}$, and (d) $|\Psi|^{2}$, for a spin-polarized spinor BEC of ${ }^{23} \mathrm{Na}$. The axes of directions are indicated in the bottom right corner. The strengths of the SOC are $V_{x}=V_{y}=V=1.5$ and the final temperature is $k_{B} T=2$. The rightmost column shows the phase profiles of equilibrium state. The final particle numbers are $N_{1} \approx 9.26 \times 10^{4}$, $N_{0} \approx 1.09 \times 10^{4}, N_{-1} \approx 1.01 \times 10^{3}$, corresponding to a magnetization ratio of $M / N \approx 0.87$.

need to consider the interacting part. It is straightforward to show that both PW and SVL states lead to the identical density-density interaction energy and thus the only difference is the spin-exchange term. As a result, the spin-exchange interaction energy per particle, $\epsilon_{\text {spin }}=g_{s} \rho \int S^{2}(\mathbf{r}) d^{2} r$, is $g_{s} \rho$ and $3 g_{s} \rho / 4$ for $\mathrm{PW}$ and SVL states, respectively, where $\rho=|\Psi|^{2}$ is the total particle density. Since $g_{s}<0$ in the current case, the SVL state has a higher energy accordingly.

The SVL state is sustained by the vorticity produced by the combining effect of SOC and thermal noises. We note that, unlike the zero-temperature Gross-Pitaevskii equation in which the total particle number $N$ is fixed, the chemical potential $\mu$ is fixed in our simulations. From the numerical results, we find that both $N$ and the number of mass vortices in $\Psi_{0}$ increase as $V$ increases. Since PW and SVL states are gapped by an amount of energy proportional to the total particle density, it is expected that there exists a threshold $V_{c}$, beyond which the SVL will barely emerge. This implies that the PW state prevails over the SVL state in the large $V$ limit. The value of $V_{c}$ can be estimated by considering a homogeneous spinor BEC, in which the core size of the mass vortex nucleating in $\Psi_{0}$ is $\xi_{0} \sim\left(\hbar^{2} / 2 m \rho_{0} g_{n}\right)^{1 / 2}$, where $\rho_{0}=\left|\Psi_{0}\right|^{2}$. The instability of SVL state occurs when the lattice constant is comparable to $\xi_{0}$, as each polar core will partially overlap with the cores of contiguous merons. Accordingly, we have $V_{c} \sim\left(2 \pi^{2} m \rho_{0} g_{n} / \hbar^{2}\right)^{1 / 2}$. By the same token, if the size of the condensate is smaller than the lattice constant, the condensate is not able to accommodate SVL, which occurs when $V<0.8$ in our numerical simulations in a trap. Note that the above derivation of $V_{c}$ is restricted to the cases of homogeneous systems at nearly zero temperature. On the other hand, one may consider that, for some $V_{c}$ the energy difference becomes much larger than the energy scale associated with thermal fluctuations at a given simulation temperature, and thus the probability of observing SVL becomes vanishingly small. However, the determination of $V_{c}$ becomes irrelevant at higher temperatures since then the SVL state can no longer be described exactly as a superposition of four momentum eigenstates at nonzero temperatures.

We now consider the spinor BEC of ${ }^{23} \mathrm{Na}\left(g_{s}>0\right)$ with SOC by using the same parameter setting in our investigations for the spinor BEC of ${ }^{87} \mathrm{Rb}$. It is found that no spin texture will be created in the condensate if the AFM spin-1 gas is unpolarized initially. This finding is consistent with that in Ref. [11]. In Fig. 9, the equilibrium density and phase profiles of a spin-polarized AFM spinor BEC are shown for the lower temperature, $k_{B} T=2$. Note that the polarization ratio is $M / N \approx 0.87$, indicating that the atoms are mainly condensed in the state $m_{F}=1$. The corresponding equilibrium spin texture for this spin-polarized state is shown in Fig. 10(a). Comparing with that of a meron, the spin texture in Fig. 10(a) is configured in a quite opposite manner; the spins in the 
(a)

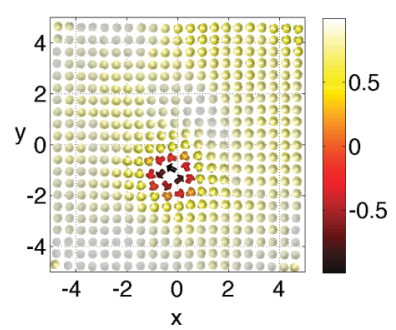

(c)



(b)



(d)



FIG. 10. (Color online) The equilibrium $\hat{\mathbf{s}}$ texture and the associated spin-density distribution $|S(\mathbf{r})|$, for different numbers of inverted merons in a spinor BEC of ${ }^{23} \mathrm{Na}$ at $k_{B} T=2$. The results for a single inverted meron are shown in (a) and (b), while those for the three inverted merons are shown in (c) and (d). The final particle numbers are $N_{1} \approx 8.32 \times 10^{4}, N_{0} \approx 1.95 \times 10^{4}, N_{1} \approx 2.21 \times 10^{3}$, corresponding to a magnetization ratio of $M / N \approx 0.77$.

outermost region always point to the $+z$ direction, while the inner ones become tilted gradually and eventually lie on the $x y$ plane, forming a circulation pattern around the polar core region within which the spin density vanishes. Such a configuration corresponds to a topological charge of $Q=-1 / 2$. In Fig. 11, the stereographic projections for a meron and the texture of Fig. 10(a) are plotted. By inverting the sphere in Fig. 11(a) and mapping the spins onto the entire plane, as shown in Fig. 11(b), we see that the same spin texture shown in Fig. 10(a) is resumed. We thus term the structure in Fig. 9(a) as the inverted meron. In Figs. 10(c)-10(d), three inverted merons with distorted cores are formed with $M / N \approx 0.77$. It is easy to verify that the single isolated inverted meron has a lower energy. Moreover, the isolated inverted meron are robust against the thermal fluctuations, which can be created in a highly polarized spin-orbit coupled AFM spinor BEC at higher temperatures, as shown in Fig. 12.

We note that the meron-like excitations in our results are intrinsic to the two-dimensional system. It would be interesting to verify whether the emergence of these fractional topological excitations is related to the Berezinskii-Kosterlitz-Thouless

(a)

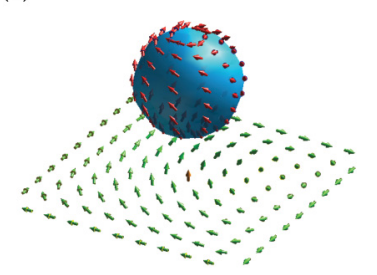

(b)

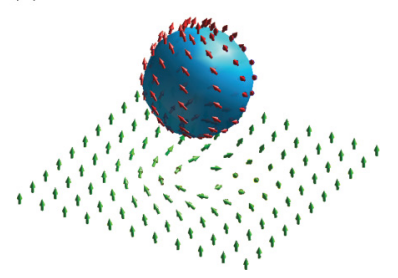

FIG. 11. (Color online) (a) Schematic plot for the stereographic projection of the spin textures onto the two-dimensional sphere $S^{2}$ for (a) meron and (b) inverted meron.

(a)

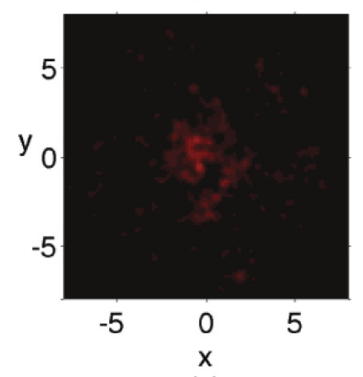

(c)



(b)

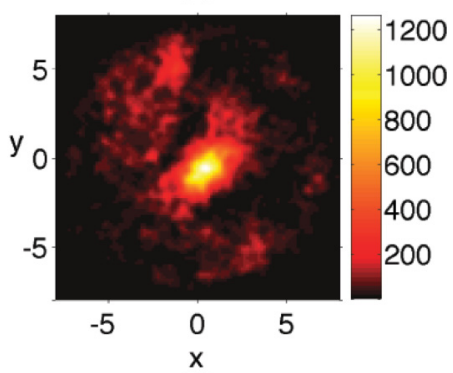

(d)



FIG. 12. (Color online) The equilibrium density profiles, (a) $\left|\Psi_{-1}\right|^{2}$, (b) $\left|\Psi_{0}\right|^{2}$, (c) $\left|\Psi_{1}\right|^{2}$, and (d) the $\hat{\mathbf{s}}$ texture, of a spinor BEC of ${ }^{23} \mathrm{Na}$ with $V_{x}=1, V_{y}=2$, and $k_{B} T=10$. The final particle numbers are $N_{1} \approx 9.06 \times 10^{4}, N_{0} \approx 1.3 \times 10^{4}, N_{1} \approx 2.24 \times 10^{3}$, corresponding to a magnetization ratio $M / N \approx 0.84$.

(BKT) transitions. Very recently, the finite-temperature properties of spin- $\frac{1}{2}$ interacting bosons with SOC in two dimensions have been studied [44]. In particular, the BKT transitions in such a system have been well characterized by drawing the analogy between the effective low-energy Hamiltonian and certain particular many-body states proposed for some well-investigated condensed-matter systems. Unfortunately, it is less likely that we can do such mappings for our more complicated model. To explore the underlying BKT physics in our current theoretical model, we may follow the approach used in [45] by identifying that what kind of topological defects can be thermally activated and proliferate near the onset of superfluid transition at finite temperatures.

\section{CONCLUDING REMARKS}

In summary, we have investigated the nonequilibrium dynamics of spin-1 BECs with SOC in the limit of rapid quench. Crystallization of merons and polar core vortices are predicted to arise in the FM spinor BEC. Likewise, isolated inverted merons can be created in the highly polarized AFM spinor BEC. Our studies provide a method to create nontrivial structure of merons and thus an opportunity to probe into the fundamental properties of skyrmionlike matter.

It is important to assume in our investigations that the spin-1 Bose gas must undergo a rapid temperature quench to enforce the formation of topological defects. In our theoretical model the dynamics has a crucial dependence on the spins, and hence a quench event is said to be "rapid" if the height of the optical dipole trap is ramped faster than the flip of a spin. In the current case, the dominating spin-related energy is the SOC, which is characterized by a frequency of $10^{3} \mathrm{~Hz}$. To fulfill a rapid quench, a ramping rate of one order higher, 
say, $10^{4} \mathrm{~Hz}$ would be required. However, a fast ramping always leads to a large loss of particles. To remedy such an undesirable consequence during the evaporative cooling, the precooling method, which enables one to capture more atoms for compensating the particle loss during the rapid quench, will be useful. Consequently, one may cool the atoms in the magnetic optical trap down to a temperature above the onset of condensation and then reload the atoms into the optical dipole trap for the rapid evaporative cooling.

\section{ACKNOWLEDGMENTS}

S.-C. Gou is supported by the National Science Council, Taiwan, under Grant No. 100-2112-M-018-001-MY3, and partly by the National Center of Theoretical Science. W. M. Liu is supported by the NSFC under Grant No. 10934010 and the NKBRSFC under Grant No. 2011CB921502. S.-C. Gou thanks Dr. Y.-J. Lin and Dr. M.-S. Chang for their helpful discussions.
[1] J. J. Sakurai, Modern Quantum Physics (Addison-Wesley, Boston, 1994).

[2] Y.-J. Lin, K. Jiménez-Garcia, and I. B. Spielman, Nature (London) 471, 83 (2011).

[3] X.-J. Liu, M. F. Borunda, X. Liu, and J. Sinova, Phys. Rev. Lett. 102, 046402 (2009).

[4] S. Bandyopadhyay, Phys. Rev. B 61, 13813 (2000).

[5] I. Žutić, J. Fabian, and S. Das Sarma, Rev. Mod. Phys. 76, 323 (2004).

[6] J. Sinova, D. Culcer, Q. Niu, N. A. Sinitsyn, T. Jungwirth, and A. H. MacDonald, Phys. Rev. Lett. 92, 126603 (2004).

[7] B. A. Bernevig, T. L. Hughes, and S.-C. Zhang, Science 314, 1757 (2006).

[8] Y. Zhang et al., Nat. Phys. 6, 584 (2010).

[9] M. Z. Hasan and C. L. Kane, Rev. Mod. Phys. 82, 3045 (2010).

[10] H. Hu, B. Ramachandhran, H. Pu, and X. J. Liu, Phys. Rev. Lett. 108, 010402 (2012).

[11] C. Wang, C. Gao, C. M. Jian, and H. Zhai, Phys. Rev. Lett. 105, 160403 (2010).

[12] Z. F. Xu, R. Lü, and L. You, Phys. Rev. A 83, 053602 (2011).

[13] T. Kawakami, T. Mizushima, and K. Machida, Phys. Rev. A 84, 011607(R) (2011).

[14] L. Jiang, X. J. Liu, H. Hu, and H. Pu, Phys. Rev. A 84, 063618 (2011).

[15] J. P. Vyasanakere and V. B. Shenoy, New J. Phys. 14, 043041 (2012).

[16] W. Yi and G.-C. Guo, Phys. Rev. A 84, 031608(R) (2011).

[17] T. W. B. Kibble, J. Phys. A 9, 1387 (1976).

[18] W. H. Zurek, Nature (London) 317, 505 (1985).

[19] L. E. Sadler et al., Nature (London) 443, 312 (2006).

[20] Chad N. Weiler et al., Nature (London) 455, 948 (2008).

[21] A. Actor, Rev. Mod. Phys. 51, 461 (1979).

[22] L. Brey, H. A. Fertig, R. Cote, and A. H. MacDonald, Phys. Rev. B 54, 16888 (1996).

[23] G. E. Brown and M. Rho, The Multifaced Skyrmion (World Scientific, Singapore, 2010).

[24] V. M. H. Ruutu, J. Kopu, M. Krusius, U. Parts, B. Placais, E. V. Thuneberg, and W. Xu, Phys. Rev. Lett. 79, 5058 (1997).
[25] R. Ishiguro et al., Phys. Rev. Lett. 93, 125301 (2004).

[26] A. E. Leanhardt, Y. Shin, D. Kielpinski, D. E. Pritchard, and W. Ketterle, Phys. Rev. Lett. 90, 140403 (2003).

[27] G. Juzeliūnas, J. Ruseckas, and J. Dalibard, Phys. Rev. A 81, 053403 (2010)

[28] J. Dalibard et al., Rev. Mod. Phys. 83, 1523 (2011).

[29] T.-L. Ho, Phys. Rev. Lett. 81, 742 (1998).

[30] T. Ohmi and K. Machida, J. Phys. Soc. Jpn. 67, 1822 (1998).

[31] T. Mizushima, N. Kobayashi, and K. Machida, Phys. Rev. A 70, 043613 (2004)

[32] A. S. Bradley, C. W. Gardiner, and M. J. Davis, Phys. Rev. A 77, 033616 (2008).

[33] S. J. Rooney, A. S. Bradley, and P. B. Blakie, Phys. Rev. A 81, 023630 (2010).

[34] S. J. Rooney, P. B. Blakie, B. P. Anderson, and A. S. Bradley, Phys. Rev. A 84, 023637 (2011).

[35] A. Sinatra et al., J. Phys. B 35, 3599 (2002).

[36] C. W. Gardiner and P. Zoller, Phys. Rev. A 58, 536 (1998).

[37] C. W. Gardiner and P. Zoller, Phys. Rev. A 61, 033601 (2000).

[38] S.-W. Su, C. H. Hsueh, I. K. Liu, T. L. Horng, Y. C. Tsai, S. C. Gou, and W. M. Liu, Phys. Rev. A 84, 023601 (2011).

[39] In principle, the isotropic SOC, $\left|V_{x}\right|=\left|V_{y}\right|$, can be realized either by tuning the phases of the laser fields or changing the geometry of the laser beams. Realistically, the fluctuations in the laser fields are liable to make $\left|V_{x}\right|$ and $\left|V_{x}\right|$ unequal. Nevertheless, we have verified numerically that SVL can survive under a small anisotropy in the SOC.

[40] T. Mizushima, K. Machida, and T. Kita, Phys. Rev. A 66, 053610 (2002).

[41] T. Kita, T. Mizushima, and K. Machida, Phys. Rev. A 66, 061601(R) (2002).

[42] T. Mizushima, K. Machida, and T. Kita, Phys. Rev. Lett. 89, 030401 (2002).

[43] N. D. Mermin et al., Phys. Rev. Lett. 36, 594 (1976).

[44] C.-M. Jian and H. Zhai, Phys. Rev. B 84, 060508 (2011).

[45] V. Pietilä, T. P. Simula, and M. Möttönen, Phys. Rev. A 81, 033616 (2010). 